
\title{
$\begin{array}{ll}\text { Research Square } & \begin{array}{l}\text { Preprints are preliminary reports that have not undergone peer review. } \\ \text { They should not be considered conclusive, used to inform clinical practice, } \\ \text { or referenced by the media as validated information. }\end{array}\end{array}$
}

\section{Daptomycin for the treatment of gram-positive periprosthetic hip infections: Can daptomycin prevent the implant removal?}

\author{
Kenichi Oe ( $\nabla$ oeken@hirakata.kmu.ac.jp ) \\ Kansai Medical University \\ Masahiro Sawada \\ Kansai Medical University \\ Hiroshi Kawamura \\ Kansai Medical University \\ Narumi Ueda \\ Kansai Medical University \\ Tomohisa Nakamura \\ Kansai Medical University

\section{Hirokazu lida} \\ Kansai Medical University \\ Takanori Saito \\ Kansai Medical University
}

\section{Research article}

Keywords: Daptomycin, Periprosthetic joint infection, Implant retention, Infection control rates, Preoperative risk scores

Posted Date: October 18th, 2019

DOI: https://doi.org/10.21203/rs.2.16186/v1

License: (c) (i) This work is licensed under a Creative Commons Attribution 4.0 International License. Read Full License

Version of Record: A version of this preprint was published at Cureus on June 22nd, 2021. See the published version at https://doi.org/10.7759/cureus.15842. 


\section{Abstract}

Background: Management of periprosthetic hip infections (PHIs) generally consists of implant removal and thorough debridement, accompanied by appropriate antibiotic therapy. Daptomycin (DAP) is a novel antibiotic, which allowed for implant retention in several patients after treating their infected joints. However, there is no consensus about implant retention or removal during treatment of PHIs. The aim of this study was to examine the effect of DAP, and to determine a surgical treatment strategy.

Methods: This study retrospectively evaluated 20 patients between August 2014 and December 2018, divided into implant retention ( $\mathrm{n}=9$ ) and implant removal groups $(n=11)$. Infection control and risk of recurrent infection were evaluated. Infection control was defined as not requiring implant removal after the final treatment. Results: Infection control rates in implant retention and implant removal groups were $67 \%$ and $90 \%$, respectively. All late chronic infections resulted in failure cases within the implant retention group. In the implant retention group, mean preoperative risk scores for successful cases were significantly higher than those for failure cases $(p<0.05)$.

Conclusions: Patients with low-risk did not require implant removal, suggesting that DAP may be a breakthrough alternative to traditional PHI management.

\section{Introduction}

The gold standard for managing periprosthetic joint infection (PJI) generally consists of implant removal and thorough debridement, accompanied by appropriate antibiotic therapy. According to the guidelines for the management of PJIs by the Infectious Diseases Society of America (IDSA) [1], the probability of implant retention is limited; only patients diagnosed with a PJI who 1) have a well-fixed prosthesis without a sinus tract and 2) are within approximately 30 days of prosthesis implantation or less than 3 weeks from the onset of infectious symptoms should be considered for debridement and retention of the prosthesis. However, there is no consensus for the treatment of PJIs regarding the choice of specific antibiotic therapy.

Daptomycin (DAP) could an option for PJI treatment because it exerts bactericidal activity against gram-positive bacteria, including multipleresistant isolates, and stationary-phase bacteria in biofilm present on implants [2]. DAP is currently available as a novel antibiotic therapy worldwide; the European Registry demonstrated high clinical success in PJI treatment with DAP therapy, including implant retention in $56 \%$ of patients [3]. However, PJI treatment with implant retention remains controversial and challenging. The aim of this study was to examine the effect of DAP on implant retention in patients with periprosthetic hip infection (PHI), and to determine a surgical treatment strategy. The hypothesis of the study was that DAP would prevent the implant removal for the patients with low-risk PHIs.

\section{Materials And Methods Study Population}

Between August 2014 and December 2018, 33 patients provided with DAP (Cubicin ${ }^{\circledR}$, MSD K. K., Tokyo, Japan) as a treatment for a PHI at our institution. DAP was administered when an infection was suspected, right after aspiration of the joint for subsequent cell culture. However, some of those patients changed the antibiotic treatment to a more appropriate one after the identification of the pathogen. In cases where no identification was made by joint aspiration, DAP therapy was initiated, and if the C-reactive protein (CRP) levels decreased, the treatment continued. Thus, a retrospective study for the treatment of infections caused by gram-positive pathogens with DAP was conducted in only 20 patients (follow-up rate of 100\%) (Fig. 1). In accordance with the IDSA guideline [1], the implant retention was initially selected, but the final decision was made intraoperatively by the surgeons. Rifampicin (RFP) was added whenever possible.

Surgical options included implant retention in 9 patients (no surgery in 4 and only debridement in 5 ) and implant removal in 11 patients (onestaged and two-staged revisions in 3 and 8, respectively). The implant retention group included 3 men and 6 women, with a mean age of 69 years (range, 36-85 years) and a mean follow-up period of 24 months (6-39 months). The mean duration of DAP therapy was 30 days (12-106 days), at a mean daily dose of $5.6 \mathrm{mg} / \mathrm{kg} /$ day $(3.8-8.3 \mathrm{mg} / \mathrm{kg} /$ day). The implant removal group included 4 men and 7 women, with a mean age of 69 years (range, 53-88 years) and a mean follow-up period of 23 months (4-50 months). The mean duration of DAP therapy was 37 days (1-60 days), at a mean daily dose of $5.8 \mathrm{mg} / \mathrm{kg} /$ day (3.8-10.0 mg/kg/day) (Table 1). The study was approved by our institutional review board. All patients provided informed consent for study participation and publication of findings.

\section{Bacterial infection diagnosis}

PHI was diagnosed according to the criteria of the Musculoskeletal Infection Society [4]. PHI was classified into four clinical categories: Type I (early postoperative infection), Type II (late chronic infection), Type III (acute hematogenous infection), and Type IV (positive intraoperative cultures) $[5,6]$. An early postoperative infection was defined as a wound infection that developed less than one month after surgery. A late 
chronic infection corresponded to an infection that developed one month or more after the index operation and that had an insidious clinical course. An acute hematogenous infection was associated with a documented or suspected antecedent bacteremia and was characterized by an acute onset of symptoms in the affected joint with the prosthesis. A patient was considered to be in the Type IV group if at least two specimens obtained at the time of revision surgery were positive on culture.

\section{Patients follow-up}

After antibiotic administration, patients were followed-up at weeks: 1, 2, 3, 4, 8, and 12, at months 6 and 9, at 1 year, and annually thereafter. Data were retrospectively analyzed by two orthopaedic surgeons who were blinded to the treatment regimens. Pathogens causing PHIs, reasons for the discontinuation of an antibiotic, and infection control rates were evaluated. Infection control was defined as the lack of clinical signs, symptom, and radiological signs of infection, a CRP level $<10 \mathrm{mg} / \mathrm{L}$ and an erythrocyte sedimentation rate $<20 \mathrm{~mm} / \mathrm{h}$. Therefore, a successful case was defined as one not requiring implant removal after treatment; failure was defined as implant removal due to recurrent infection.

\section{Clinical parameters}

For the laboratory assessment, CRP levels (mg/L) were investigated. Furthermore, the risk of recurrent infection was evaluated using the scoring system [7] (Fig. 2), based on six parameters: 1) general condition, 2) duration of infection, 3) wound complication, 4) presence of microorganisms, 5) CRP levels, and 6) necessity for bone grafting. Each parameter was rated from 0 to 2 points, giving a maximum score of 12 points for low-risk.

\section{Statistical Analysis}

Two-group comparison were conducted using Student $t$ test or Mann-Whitney $U$ test. To compare qualitative variables, Fisher exact test was applied. The Wilcoxon signed-rank test was used to compare differences in parameters before and after patient treatment. Statistical significance was defined as $p<0.05$. All analyses were performed using SAS 9.2 (SAS Institute Inc., Cary, NC, USA).

\section{Results}

The summary of results for each patient is shown in Table 2. In one patient (Case 14), DAP was discontinued because of an anaphylactic shock. Infection control rates in the implant retention and implant removal groups were $67 \%(6 / 9)$ and $90 \%$ (9/10), respectively. Clinical success rates of the implant removal group were significantly higher than those of the implant retention group $(p<0.05)$. In the implant retention group, successful cases experienced Type I (3 patients) and Type III (3 patients) infections, whereas failure cases only presented Type II infections (3 patients). Mean daily dose of DAP (mg/kg/day) in the implant retention group was 5.0 (3.8-5.9) and 7.1 (5.9-8.3) for successful and failure cases, respectively. Similarly, in the implant removal group, DAP daily dose was 5.9 (3.8-10.0) and 5.8 for successful and failure cases, respectively.

The mean CRP values for each group were recorded for 8 weeks, and the results were grouped according to the outcome of the DAP treatment (success of failure) for each group (Fig. 3). CRP levels in all cases, except for the failure case in the implant removal group, were significantly decreased. Mean preoperative risk scores of the implant retention group were 7.8 points (7-9 points) and 6.3 points (6-7 points) for successful and failure cases, respectively. Mean preoperative risk scores for successful cases were significantly higher than those for failure cases ( $p<$ 0.05). In the implant removal group, the score for successful and failure cases were 7.3 points (6-10 points) and 5.0 points, respectively.

Additionally, antibiotic conversion to DAP was observed in some cases. In one patient (Case 12), one-staged revision was performed because of acetabular cup loosening and dislocation due to PHI. After DAP therapy, the treatment continued its normal course (Figs. 4, 5).

\section{Discussion}

The frequency of PJIs is increasing, according to the Nordic Arthroplasty Register Association [8], and the treatment of PJIs should target grampositive pathogens because such organisms cause the majority of PJIs, and especially those that became antibiotic resistant [9-12]. In Japan, the proportion of gram-positive PJIs is over $70 \%$, while $42 \%$ of those are methicillin-resistant Staphylococcus aureus (MRSA) [9]. In Europe and the United States, more than $50 \%$ of PJIs are typically caused by staphylococcal organisms; and this percentage value is expected to increase $[11,12]$. The increased frequency of gram-positive infections and the rise in resistance to commonly used antibiotics have led to the need of novel antibiotic therapies, such as DAP, which has high antimicrobial penetration into biofilms with low bactericidal concentration [13]. According to the European Registry, DAP was effective and safe in patients with osteomyelitis or those with orthopedic device infections; it was also a 
valuable treatment option for the management of gram-positive infections $[3,14]$. However, in the treatment of PJIs by using DAP, there is no consensus whether the implant should be removed or not.

In the current study, the clinical success rates in the implant removal group were significantly higher than those in the implant retention group. However, the patients without chronic late infection and with a score higher than 7 points did not require implant removal. Tsukayama et al. [5] analyzed the relationship between clinical settings and outcomes in 106 patients with PHIs, and reported that all patients with early postoperative infections (success rates: $71 \%$ ) or acute hematogenous infection (success rates: $50 \%$ ) had only debridement, while all patients with late chronic infection (success rates: 85\%) were subjected to revision arthroplasty. Therefore, patients with late chronic infections may not be approved for implant retention even if DAP is administered.

Past reports of DAP treatment of PJI are shown in Table 3, and even some authors recommended the use of high-dose of DAP for the treatment of PJI with implant retention [2,15-21]. Furthermore, the European Registry demonstrated high clinical success with DAP therapy, including both implant retention (56\%) and removal (44\%). Additionally, patients receiving both DAP and RFP showed higher success rates than those who did not concomitantly receive RFP [3]. Interestingly, in vitro experiments showed that DAP had the fastest eradication rate for MRSA embedded in a biofilm; therefore, the combination of DAP and RFP may be a promising treatment option for implant-associated MRSA infections [22, 23]. Moreover, the combination of high-dose DAP (equivalent to $8-10 \mathrm{mg} / \mathrm{kg} /$ day in humans) and RFP was highly effective for the treatment of foreign body-related MRSA infections [24, 25]. Lora-Tamayo et al. [19] also analyzed 18 staphylococcal PJls in a multicenter study and concluded that high-dose DAP (10 mg/kg/day) plus RFP was a good initial treatment for PJls with implant retention. In the current study, however, the mean daily dose of DAP for successful cases of implant retention was not always high, although RFP was administered whenever possible. Either way, RFP may be an important addition to consider when treating PJIs with DAP, with or without implant removal.

Some limitations of our study must be noted. First, the sample size was small, involving only 20 individuals, due to the difficulties of obtaining a larger patient sample from a single institution. Furthermore, the current study was not performed as a randomized controlled trial. Patients with refractory PHIs required treatment on a case-by-case basis; therefore, the optimal surgical intervention and daily DAP dose also differed. Second, some patients, who did not undergo implant removal after treatment, continued to take other antibiotics orally. As a consequence, because they did not undergo implant removal, they may have actually experienced recurrent infections. Third, we administered DAP when a PHI was suspected prior to the identification of the specific pathogen, because such organisms cause the majority of PHIs and DAP still works in presence of antibiotic resistance. However, such approach may lead to administration of excessive levels of antibiotics if treatments need to be switched to a more appropriate antibiotic after pathogen identification.

\section{Conclusion}

Infection control rates in the implant retention and implant removal groups were $67 \%$ and $90 \%$, respectively. Late chronic infection was the infection type for all the failure cases in the implant retention group. Furthermore, in the implant retention group, the mean preoperative risk score for the successful cases was significantly higher than that for the failure cases. Patients with low-risk, who did not present chronic late infection but received a preoperative risk score higher than 7 points, may not require implant removal, suggesting that DAP may be a breakthrough alternative to traditional PHI management.

\section{Declarations}

\section{Abbreviations}

CRP: C-reactive protein; DAP: Daptomycin; IDSA: Infectious Diseases Society of America; MRSA: Methicillin-resistant Staphylococcus aureus; PHI: Periprosthetic hip infection; PJI: Periprosthetic joint infection; RFP: Rifampicin

\section{Acknowledgements}

Not applicable

\section{Authors' contributions}

KO designed the study. MS, HK, and NU collected and performed the analysis of the data.

KO wrote the manuscript. TN, HI, and TS supervised the study. All authors read and approved the final manuscript.

\section{Funding}


This research did not receive any specific funding from public or commercial entity.

\section{Available of data and materials}

All data generated or analyzed during this study are included in this published article.

\section{Ethics approval and consent to participate}

As stated in the Methods section, Ethical approved of the study was provided by the Institutional Review Board (Kansai Medical University, date 15/07/2015, No.H160737). All participants were informed of the study and signed a written informed consent. The study was conducted according to the ethical principles stated in the Declaration of Helsinki.

\section{Consent for publication}

We received written informed consent from the patients.

\section{Competing interests}

The authors declare that they have no competing interests.

\section{References}

1. Osmon DR, Berbari EF, Berendt AR, et al. Infectious Diseases Society of America. Diagnosis and management of prosthetic joint infection: clinical practice guidelines by the Infectious Diseases Society of America. Clin Infect Dis. 2013;56(1):e1-

2. Rao N, Regalla DM. Uncertain efficacy of daptomycin for prosthetic joint infections: a prospective case series. Clin Orthop Relat Res. $2006 ; 451: 34-7$.

3. Malizos K, Sarma J, Seaton RA, et al. Daptomycin for the treatment of osteomyelitis and orthopaedic device infections: real-world clinical experience from a European registry. Eur J Clin Microbiol Infect Dis. 2016;35(1):111-8.

4. Parvizi J, Zmistowski B, Berbari EF, et al. New definition for periprosthetic joint infection: from the Workgroup of the Musculoskeletal Infection Society. Clin Orthop Relat Res. 2011;469:2992-4.

5. Tsukayama DT, Estrada R, Gustilo RB. Infection after total hip arthroplasty. A study of the treatment of one hundred and six infections. J Bone Joint Surg Am. 1996;78(4):512-

6. CuiQ, Mihalko WM, Shields JS, Ries M, Saleh KJ. Antibiotic-impregnated cement spacers for the treatment of infection associated with total hip or knee arthroplasty. J Bone Joint Surg Am. 2007;89(4):871-

7. Oe K, Iida H, Ueda N, Nakamura T, Okamoto N, Ueda Y. Pre-operative scoring system to determine the surgical strategy for periprosthetic hip infection. Int Orthop. 2015;39(1):19-

8. Dale H, Fenstad AM, Hallan G, et al. Increasing risk of prosthetic jointinfection after total hip arthroplasty. Acta Orthop. 2012;83(5):449-

9. Yamamoto K, Masaoka T, Ishii Y, et al. Epidemiology of surgical site infection following implant surgery. Orthopaedic Surgery and Traumatology. 2010;53(5):419-25 [In Japanese].

10. Tande AJ, Patel R. Prosthetic joint infection. Clin Microbiol Rev. 2014;27(2):302-

11. Aggarwal VK, Bakhshi H, Ecker NU, Parvizi J, Gehrke T, Kendoff D. Organism profile inperiprosthetic joint infection: pathogens differ at two arthroplasty infection referral centers in Europe and in the United States. J Knee Surg. 2014;27(5):399-

12. Kapadia BH, Berg RA, Daley JA, Fritz J, Bhave A, Mont MA. Periprostheticjoint infection. 2016;387(10016):386-94.

13. Tiemersma EW, Bronzwaer SL, Lyytikäinen O, et al. European Antimicrobial Resistance Surveillance System Participants. Methicillin-resistant Staphylococcus aureus in Europe, 1999-2002. Emerg Infect Dis. 2004;10(9):1627-

14. Gonzalez-RuizA, Gargalianos-Kakolyris P, Timerman A, et al. Daptomycin in the Clinical Setting: 8-Year Experience with Gram-positive Bacterial Infections from the EU-CORE(SM) Registry. Adv Ther. 2015;32(6):496-

15. Antony SJ, Tiscareno-Grajeda I, Misenhiemer G, Heyderman J. Use of daptomycin in the treatment of prosthetic joint infections: A prospective observational study of 30 patients with infected prosthetic joint infections. The internet journal of infection disease. 2008;7:1- 
16. Licitra CM, Crespo A, Licitra D, Wallis-Crespo M. Daptomycin for the treatment of osteomyelitis and prosthetic joint infection: Retrospective analysis of efficacy and safety in an outpatient infusion center. The internet journal of infection disease. 2010;9:1-

17. Corona Pérez-Cardona PS, Barro Ojeda V, Rodriguez Pardo D, et al. Clinical experience with daptomycin for the treatment of patients with knee and hip periprosthetic joint infections. J Antimicrob Chemother. 2012;67(7):1749-

18. JugunK, Vaudaux P, Garbino J, Pagani L, Hoffmeyer P, Lew D, Uçkay I. The safety and efficacy of high-dose daptomycin combined with rifampicin for the treatment of Gram-positive osteoarticular infections. Int Orthop. 2013;37(7):1375-

19. Lora-TamayoJ, Parra-Ruiz J, Rodríguez-Pardo D, et al. High doses of daptomycin $(10 \mathrm{mg} / \mathrm{kg} / \mathrm{d})$ plus rifampin for the treatment of staphylococcal prosthetic joint infection managed with implant retention: a comparative study. Diagn Microbiol Infect Dis. 2014;80(1):66-

20. Kuo FC, Yen SH, Peng KT, Wang JW, Lee MS. Methicillin-resistant Staphylococcal periprosthetic joint infections can be effectively controlled by systemic and local daptomycin. BMC Infect Dis. 2016;16:48.

21. Chang YJ, Lee MS, Lee CH, Lin PC, Kuo FC. Daptomycin treatment in patients with resistant staphylococcal periprosthetic joint infection. BMC Infect Dis. 2017;17:736.

22. RaadI, Hanna H, Jiang Y, Dvorak T, Reitzel R, Chaiban G. Comparative activities of daptomycin, linezolid, and tigecycline against catheter-related methicillin-resistant Staphylococcus bacteremic isolates embedded in biofilm. Antimicrob Agents Chemother. 2007;51(5):1656-

23. John AK, Baldoni D, Haschke M, et al. Efficacy of daptomycin in implant-associated infection due to methicillin-resistant Staphylococcus aureus: importance of combination with rifampin. Antimicrob Agents Chemother. 2009;53(7):2719-

24. Garrigós C, Murillo O, Euba G, et al. Efficacy of usual and high doses of daptomycin in combination with rifampin versus alternative therapies in experimental foreign-body infection by methicillin-resistant Staphylococcus aureus. Antimicrob Agents Chemother. 2010;54(12):5251-

25. Saleh-MghirA, Muller-Serieys C, Dinh A, Massias L, Crémieux AC. Adjunctive rifampin is crucial to optimizing daptomycin efficacy against rabbit prosthetic joint infection due to methicillin-resistant Staphylococcus aureus. Antimicrob Agents Chemother. 2011;55(10):4589-

\section{Tables}

Table 1 Preoperative patient characteristics

\begin{tabular}{l|c|c|c|}
\hline cteristics & Implant retention & Implant removal & $\boldsymbol{p}$ value \\
\hline er of joints & 9 & 11 & \\
\hline age at surgery, years (range) & $69(36-85)$ & $69(53-88)$ & $0.580^{\mathrm{a}}$ \\
\hline r, male:female & $3: 6$ & $4: 7$ & $0.630^{\mathrm{b}}$ \\
\hline follow-up period, months (range) & $24(6-39)$ & $23(4-50)$ & $0.061^{\mathrm{a}}$ \\
\hline er of patients previously administered with other antibiotics & 7 & 9 & $0.625^{\mathrm{b}}$ \\
\hline dose, mg/kg/day (range) & $5.8(3.8-8.3)$ & $5.8(3.8-10.0)$ & $0.313^{\mathrm{a}}$ \\
\hline duration of daptomycin therapy, day (range) & $30(12-106)$ & $37(1-60)$ & $0.095^{\mathrm{c}}$ \\
\hline er of patients administered with rifampicin & 7 & 8 & $0.604^{\mathrm{b}}$ \\
\hline ad implant & & & $0.579^{\mathrm{b}}$ \\
lar hip arthroplasty & 1 & 2 & \\
I hip arthroplasty & 8 & 9 & \\
\hline :al intervention during this therapy, number & & & \\
? & 5 & 0 & $0.026^{\mathrm{b}}$ \\
debridement & 5 & 3 & $0.008^{\mathrm{b}}$ \\
staged revision & 0 & 8 & $0.145^{\mathrm{b}}$ \\
staged revision & 0 & $0.001^{\mathrm{b}}$ \\
\hline
\end{tabular}

${ }^{\mathrm{a}}$ Student $t$-test, ${ }^{\mathrm{b}}$ Fisher exact test, ${ }^{\mathrm{c}}$ Mann-Whitney $U$ test

Table 2 Characteristics and results of each patient infected with gram-positive pathogens before and after DAP treatment 


\begin{tabular}{|c|c|c|c|c|c|c|c|c|c|}
\hline$\overline{\mathrm{ase}}$ & Age/Gender & $\begin{array}{l}\text { Infected } \\
\text { implant }\end{array}$ & Pathogen & $\begin{array}{c}\text { Infection } \\
\text { type }^{\mathrm{a}}\end{array}$ & $\begin{array}{c}\text { Duration } \\
\text { of DAP } \\
\text { (day) }\end{array}$ & $\begin{array}{c}\text { DAP daily } \\
\text { dose } \\
\text { (mg/kg/day) }\end{array}$ & $\begin{array}{c}\text { Preoperative } \\
\text { scoring }\end{array}$ & $\begin{array}{c}\text { Surgical } \\
\text { intervention }\end{array}$ & $\begin{array}{l}\text { Additional } \\
\text { surgery or } \\
\text { adverse } \\
\text { event }\end{array}$ \\
\hline 1 & $81 /$ female & THA & \multirow{20}{*}{ 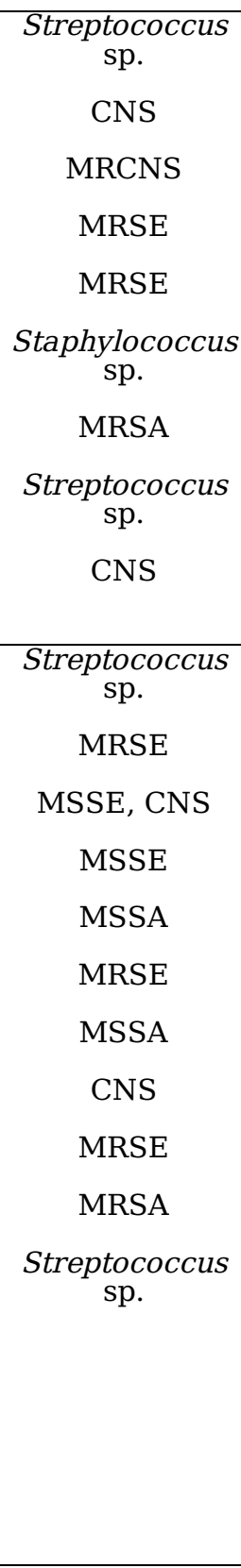 } & III & 15 & 4.2 & 9 & No surgery & $(-)$ \\
\hline 2 & 75/female & THA & & III & 16 & 5.1 & 8 & No surgery & $(-)$ \\
\hline 3 & 77/female & BHA & & I & 12 & 5.3 & 7 & No surgery & $(-)$ \\
\hline 4 & $62 /$ female & THA & & II & 14 & 8.3 & 7 & No surgery & Two-staged \\
\hline 5 & $36 /$ male & THA & & I & 27 & 5.9 & 8 & Only & IEVISIOII \\
\hline 6 & $64 /$ male & THA & & III & 36 & 5.7 & 8 & 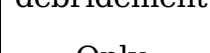 & $(-)$ \\
\hline 7 & $85 /$ male & THA & & I & 27 & 3.8 & 7 & debridement & (-) \\
\hline 8 & 64/female & THA & & II & 19 & 5.9 & 6 & Only & $T$ Therom \\
\hline 9 & 75/female & THA & & II & 106 & 7.0 & 6 & $\begin{array}{l}\text { Only } \\
\text { debridement } \\
\text { Only } \\
\text { debridement }\end{array}$ & $\begin{array}{l}\text { revision } \\
\text { Two-staged } \\
\text { revision }\end{array}$ \\
\hline 10 & 76/female & THA & & II & 49 & 7.6 & 10 & One-staged & $(-)$ \\
\hline 11 & 88/male & BHA & & III & 22 & 5.3 & 8 & & $(-)$ \\
\hline 12 & $82 /$ female & THA & & II & 31 & 3.8 & 7 & revision & $(-)$ \\
\hline 13 & $62 /$ female & THA & & I & 42 & 6.0 & 8 & One-staged & $(-)$ \\
\hline 14 & 55/female & THA & & I & 1 & 4.4 & 8 & Trustal & Anaphylaxis \\
\hline 15 & 66/male & BHA & & I & 60 & 4.5 & 7 & revision & $(-)$ \\
\hline 16 & 53/male & THA & & II & 25 & 4.9 & 7 & Two-staged & $(-)$ \\
\hline 17 & $62 /$ male & THA & & II & 31 & 5.0 & 7 & & $(-)$ \\
\hline 18 & $65 /$ female & THA & & II & 57 & 10.0 & 6 & revision & $(-)$ \\
\hline 19 & 74/female & THA & & II & 48 & 5.8 & 6 & Two-staged & $(-)$ \\
\hline 20 & 74/female & THA & & II & 44 & 5.8 & 5 & $\begin{array}{c}\text { Two-staged } \\
\text { revision } \\
\begin{array}{c}\text { Two-staged } \\
\text { revision }\end{array} \\
\begin{array}{c}\text { Two-staged } \\
\text { revision }\end{array} \\
\begin{array}{c}\text { Two-staged } \\
\text { revision }\end{array}\end{array}$ & $\begin{array}{l}\text { Two-staged } \\
\text { revision }\end{array}$ \\
\hline
\end{tabular}

anfection type: Type I (early postoperative infection), Type II (late chronic infection), Type III (acute hematogenous infection), and Type IV (positive intraoperative cultures)

DAP daptomycin, THA total hip arthroplasty, BHA bipolar hip arthroplasty, CNS coagulase-negative Staphylococcus, MRCNS methicillin-resistant coagulase-negative Staphylococcus, MRSE methicillin-resistant Staphylococcus epidermidis, MRSA methicillin-resistant Staphylococcus aureus, MSSE methicillin-sensitive Staphylococcus epidermidis, MSSA methicillinsensitive Staphylococcus aureus

Table 3 Past reports of daptomycin treatment of periprosthetic joint infections 


\begin{tabular}{|c|c|c|c|c|c|}
\hline \multirow[t]{2}{*}{ Author } & \multirow{2}{*}{$\begin{array}{c}\text { Year } \\
\text { published }\end{array}$} & \multirow{2}{*}{$\begin{array}{l}\text { Number of } \\
\text { joints }\end{array}$} & \multirow{2}{*}{$\begin{array}{c}\text { Daily dose of daptomycin } \\
\text { (mg/kg/day) }\end{array}$} & \multicolumn{2}{|c|}{ Infection control rates } \\
\hline & & & & $\begin{array}{l}\text { Implant } \\
\text { retention }\end{array}$ & $\begin{array}{l}\text { Implant } \\
\text { removal }\end{array}$ \\
\hline Rao et al. [2] & 2006 & 11 & 4 & $25 \%(1 / 4)$ & $71 \%(5 / 7)$ \\
\hline Antony et al. [15] & 2008 & 30 & 6 & none & $67 \%(20 / 30)$ \\
\hline Licitra et al. [16] & 2010 & 14 & $\geq 6$ & $100 \%(3 / 3)$ & $100 \%(11 / 11)$ \\
\hline Corona Pérez-Cardona et & 2012 & 14 & 6.6 & $100 \%(5 / 5)$ & $67 \%(6 / 9)$ \\
\hline di. $[1 /]$ & 2013 & 13 & $\geq 8$ & $100 \%(4 / 4)$ & $100 \%(9 / 9)$ \\
\hline Lon & 2014 & 18 & 10 & $50 \%(9 / 18)$ & none \\
\hline Lora-1 amayo et al. [19] & 2016 & 22 & 6 & none & $100 \%(22 / 22)$ \\
\hline$[20 \mathrm{O}$ & 2017 & 16 & 8.3 & $80 \%(4 / 5)$ & $91 \%(10 / 11)$ \\
\hline Current study & & 19 (Hip) & 5.8 & $67 \%(6 / 9)$ & $90 \%(9 / 10)$ \\
\hline
\end{tabular}

Figures

\section{Suspected PHI: Aspirate culture}

33 cases between August 2014 and December 2018

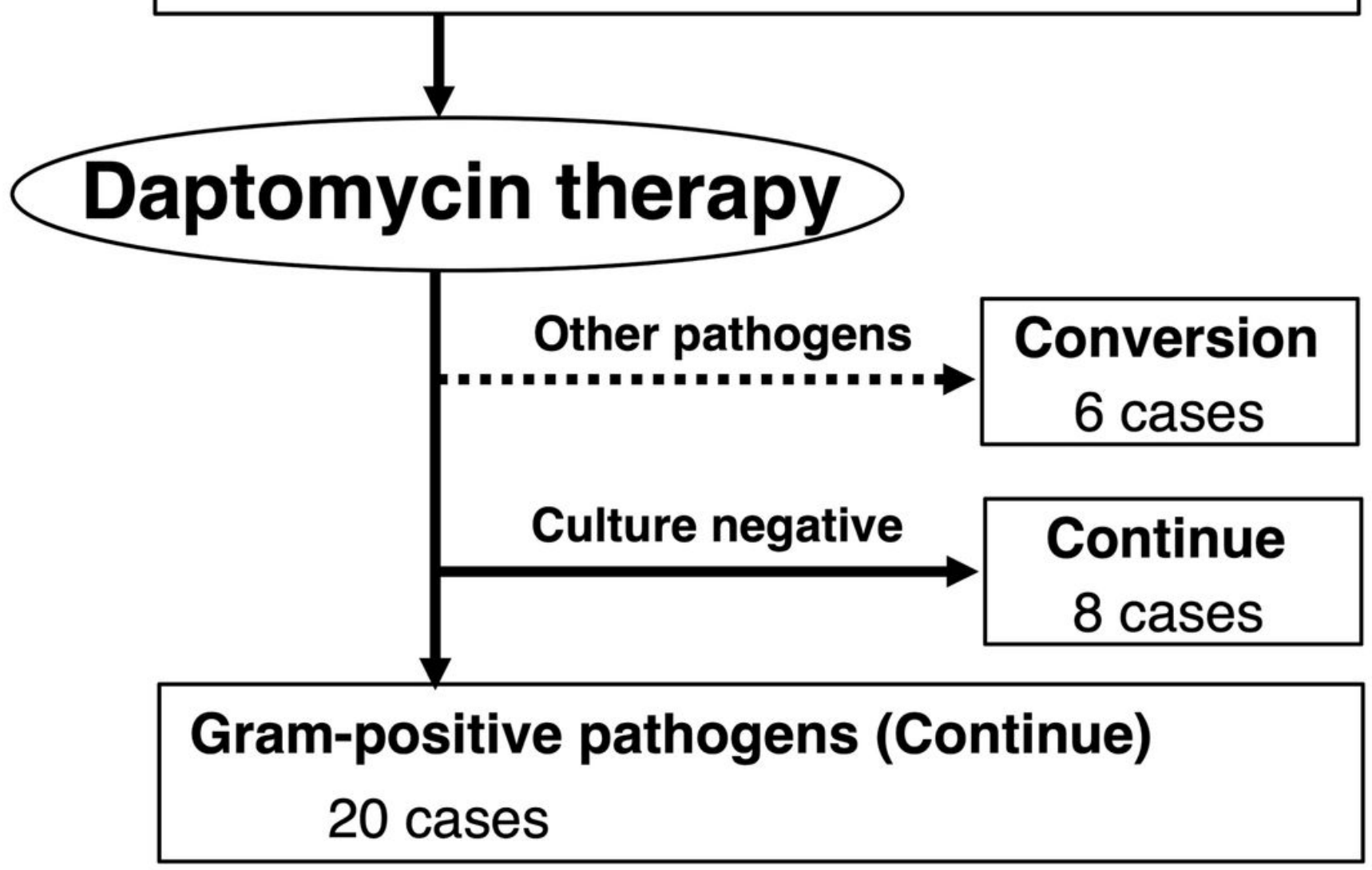

Figure 1

Study flowchart. PHI, periprosthetic hip infection 
1. General condition

Poor (American Society of Anesthesiologists's class $\geq 3$, etc.)

Moderate (Diabetes Mellitus, Steroid, Autoimmune disease, etc.)

Good

2. Duration of infection, Number of past operations

Past operations $\geq 2$ (including Osteotomy, Debridement, etc.) 0

Late infection 1

Early or Delayed infection 2

3. Wound complication after initial operation

Sinus tract, Abscess

Slightly damaged (reddish, local heat, etc.) Intact

4. Presence of microorganism

Organism resistance to the antibiotics, Gram-negative, MRSA or MRSE $\quad 0$ Unknown

Organism sensitive to the antibiotics

C-reactive protein $(\mathrm{mg} / \mathrm{L})$

$>50$

50-5

$<5$

6. Necessity for bone grafting

Necessity

Unnecessity

Figure 2

Pre-operative scoring system to assess the risk of recurrent infection. Each parameter was rated from 0 to 2 points, giving a maximum score of 12 points for low-risk 
$(\mathrm{mg} / \mathrm{L})$

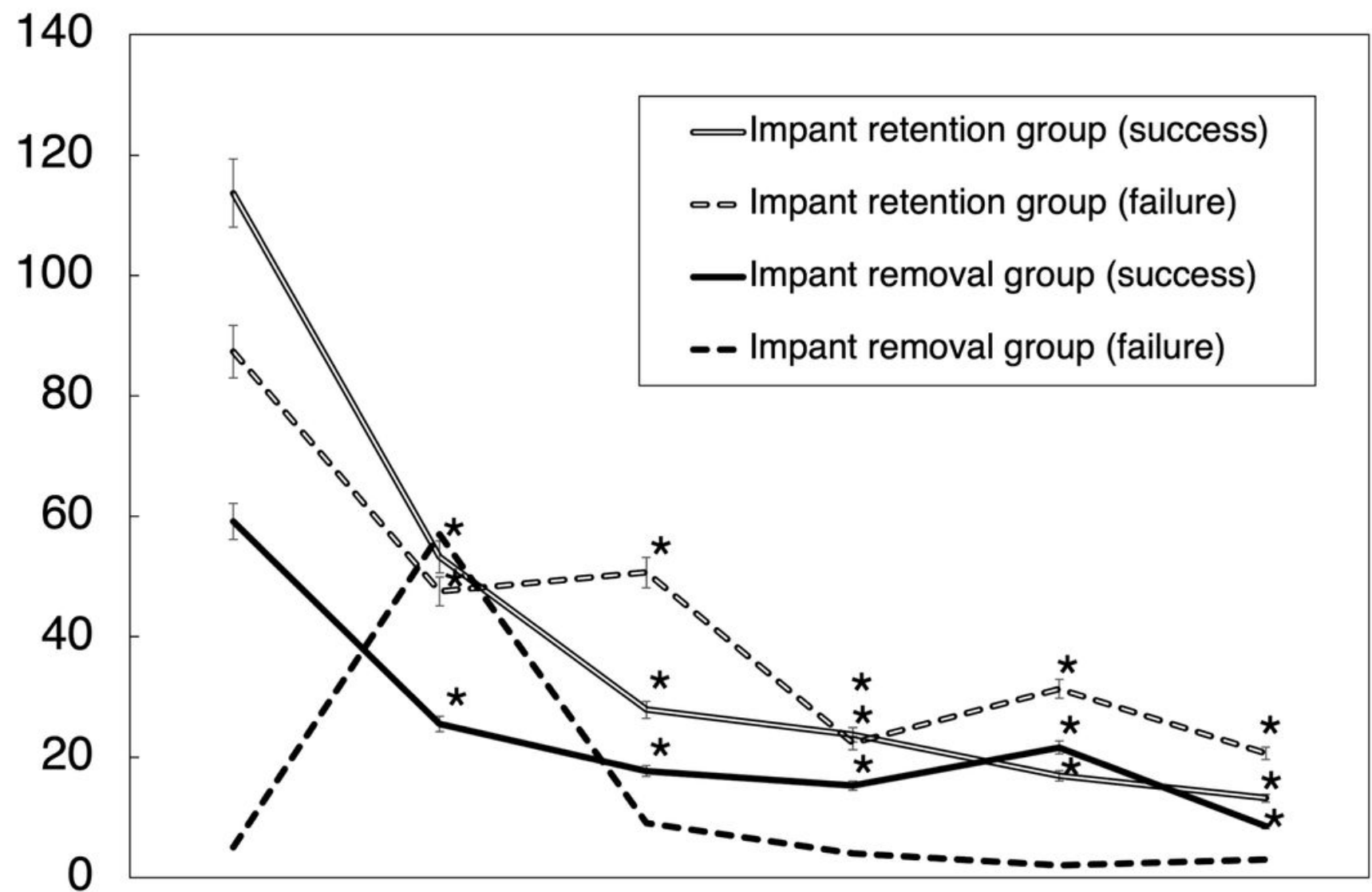

week 0 week 1 week 2 week 3 week 4 week 8

$\left({ }^{*} p<0.05\right.$ : Wilcoxon signed-rank test)

Figure 3

Mean C-reactive protein concentration in patients infected with Gram-positive bacteria and treated with daptomycin. The Wilcoxson signed-rank test was used to compare differences before and after treatment of patients. Data are expressed as the means and two-sided $95 \%$ confidence interval 

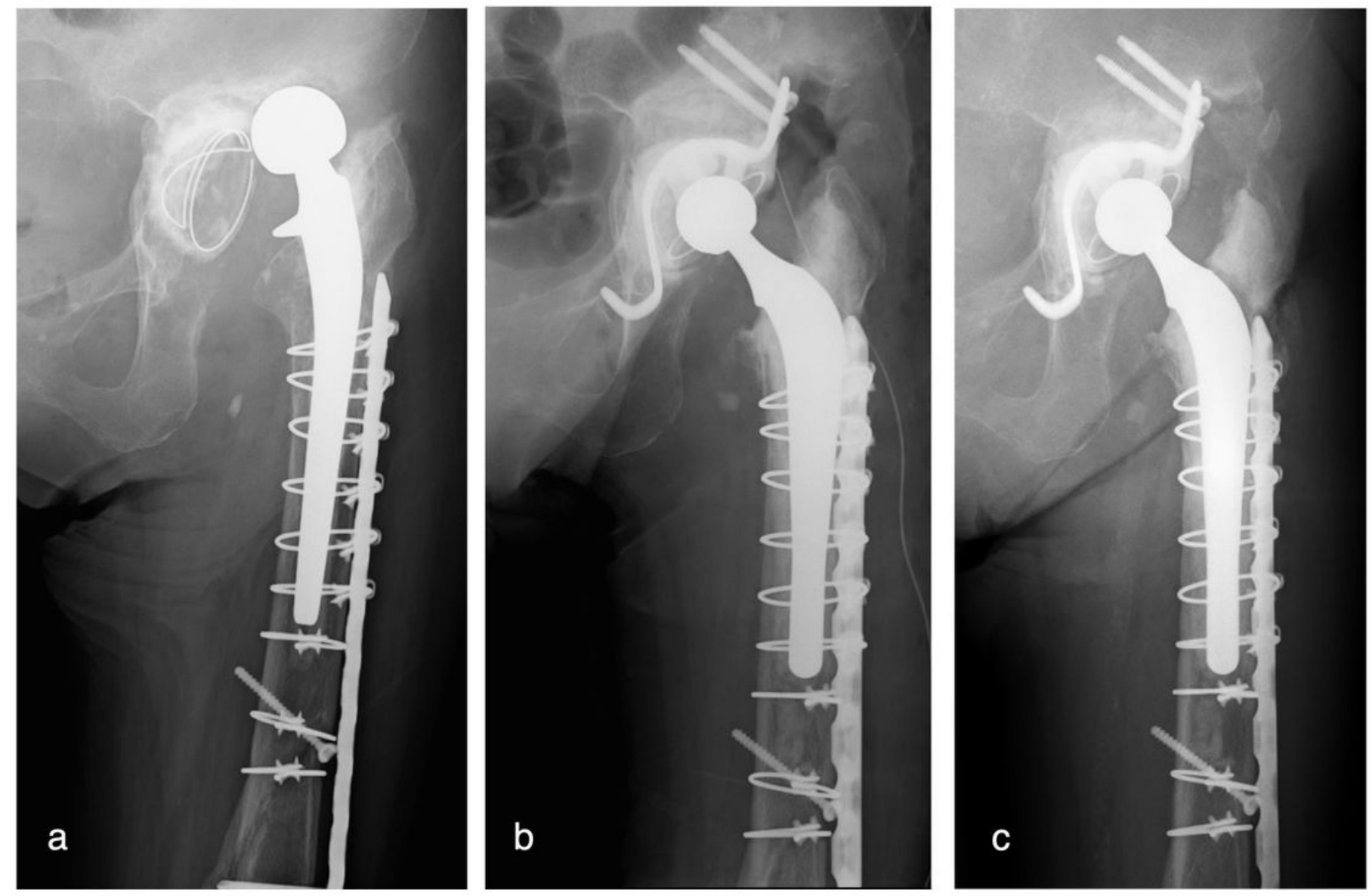

\section{Figure 4}

Anteroposterior hip radiographs of an 82-year-old woman who underwent primary THA 14 years ago and revision THA 1 year ago, with rheumatoid arthritis and hemodialysis (Case 12, preoperative score of 7 points). a Radiograph showing acetabular cup loosening and dislocation due to periprosthetic hip infection. b Radiograph immediately after one-staged revision THA, including cup and stem. c Radiograph at 4 years postoperatively 
(mg/L)

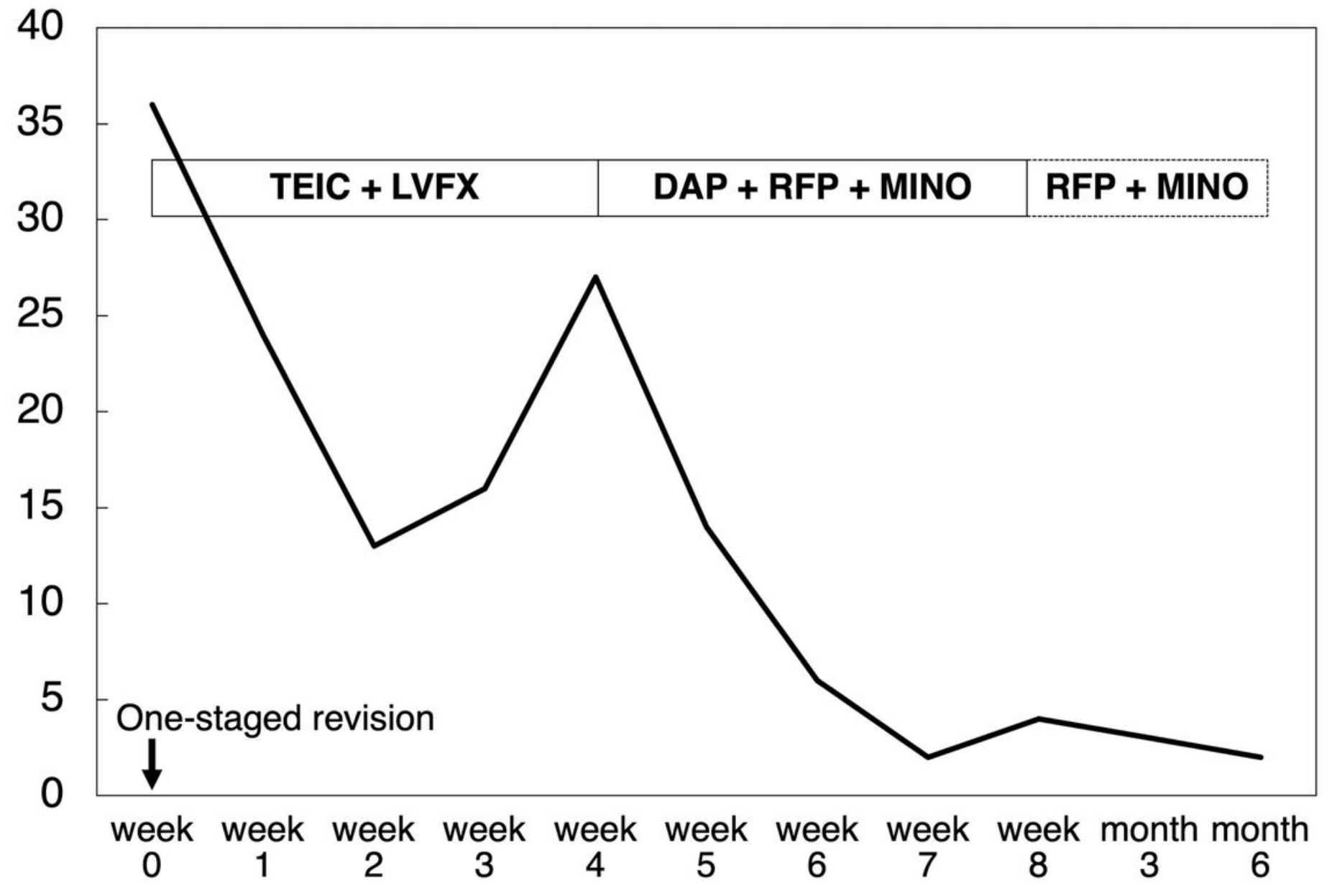

Figure 5

Treatment course and C-reactive protein concentration after DAP therapy in Case 12. TEIC, teicoplanin; LVFX, levofloxacin; DAP, daptomycin; RFP, rifampicin; MINO, minocycline hydrochloride 